\title{
A case of local recurrence and distant metastasis following curative endoscopic submucosal dissection of early gastric cancer
}

\author{
Seiichiro Abe $\cdot$ Ichiro Oda $\cdot$ Takeshi Nakajima $\cdot$ Haruhisa Suzuki $\cdot$ \\ Satoru Nonaka $\cdot$ Shigetaka Yoshinaga $\cdot$ Shigeki Sekine $\cdot$ Hirokazu Taniguchi $\cdot$ \\ Ryoji Kushima $\cdot$ Satoru Iwasa $\cdot$ Yutaka Saito $\cdot$ Hitoshi Katai
}

Received: 8 October 2013/ Accepted: 30 December 2013/Published online: 31 January 2014

(c) The International Gastric Cancer Association and The Japanese Gastric Cancer Association 2014

\begin{abstract}
Currently in Japan, differentiated-type gastric submucosal invasive cancers $<500 \mu \mathrm{m}$ with negative lymphovascular involvement are included in expanded pathological criteria for curative endoscopic treatment. This categorization is based on a retrospective examination of surgical resection cases in which patients suitable for such expanded criteria were determined to have a negligible risk of lymph node metastasis. We performed endoscopic submucosal dissection on a 66-year-old man with early gastric cancer in June 2004, and pathology revealed a well-differentiated adenocarcinoma, $16 \times 8 \mathrm{~mm}$ in size, minute submucosal invasion depth $(100 \mu \mathrm{m})$, and negative lymphovascular invasion or ulceration as well as tumorfree margins, so the case was diagnosed as a curative resection. In this case, however, local recurrence and distant metastasis resulted in August 2011. The patient received systemic chemotherapy but died of gastric cancer 23 months after recurrence.
\end{abstract}

S. Abe $(\bowtie) \cdot$ I. Oda $\cdot$ T. Nakajima $\cdot$ H. Suzuki $\cdot$ S. Nonaka S. Yoshinaga $\cdot$ Y. Saito

Endoscopy Division, National Cancer Center Hospital, 5-1-1

Tsukiji, Chuo-ku, Tokyo 104-0045, Japan

e-mail: seabe@ncc.go.jp

S. Sekine $\cdot$ H. Taniguchi $\cdot$ R. Kushima

Pathology Division, National Cancer Center Hospital, Tokyo, Japan

S. Iwasa

Gastrointestinal Oncology Division, National Cancer Center

Hospital, Tokyo, Japan

H. Katai

Gastric Surgery Division, National Cancer Center Hospital,

Tokyo, Japan
Keywords Early gastric cancer - Endoscopic submucosal dissection · Expanded criteria $\cdot$ Local recurrence $\cdot$ Distant metastasis

\section{Introduction}

Endoscopic submucosal dissection (ESD) has become widely used as a minimally invasive treatment for early gastric cancer (EGC) that has negligible lymph node metastasis. The expanded pathological criteria of endoscopic treatment based on retrospective examinations of surgical resection cases had been accepted [1-3]. However, we experienced for the first time a case of local recurrence and distant metastasis following ESD resulting in a pathologically curative resection of minute submucosal (SM1, $<500 \mu \mathrm{m}$ from muscularis mucosa) gastric cancer.

\section{Case report}

A 66-year-old man was admitted for the treatment of EGC in June 2004. There was no medical history of other malignant tumors. The lesion was preoperatively diagnosed as a type 0-IIa well-differentiated adenocarcinoma limited to mucosa, $20 \mathrm{~mm}$ in size, with an ulcer scar on the lesser curvature of the middle gastric body (Fig. 1a). ESD was performed on this lesion, resulting in an en bloc resection. Pathological examination of 2-mm-interval slices from the entire specimen revealed well-differentiated adenocarcinoma, $16 \times 8 \mathrm{~mm}$ in size, SM1 invasion depth $(100 \mu \mathrm{m}$ from muscularis mucosa), and negative lymphovascular invasion or ulceration and no undifferentiated-type component as well as tumor-free margins (Fig. 1b). This lesion 


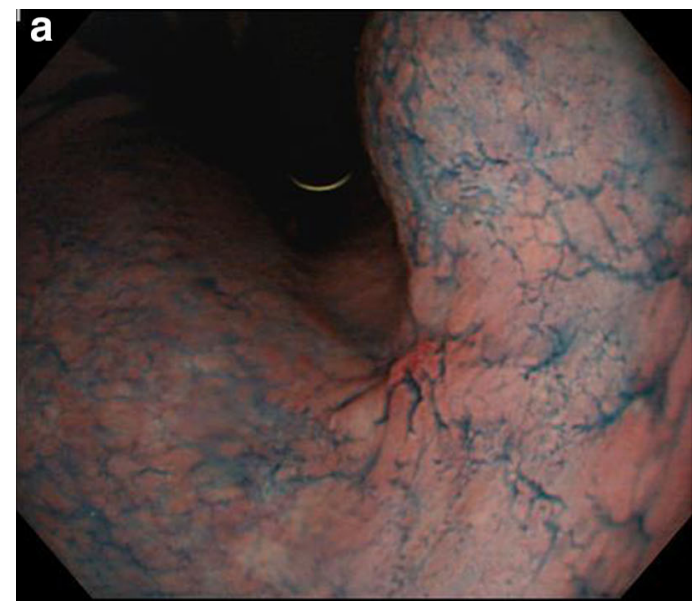

Fig. 1 a Esophagogastroduodenoscopy detected a type 0-IIa lesion $20 \mathrm{~mm}$ in size with ulcer scar on the lesser curvature of the middle gastric body in June 2004. b Pathological examination revealed a type

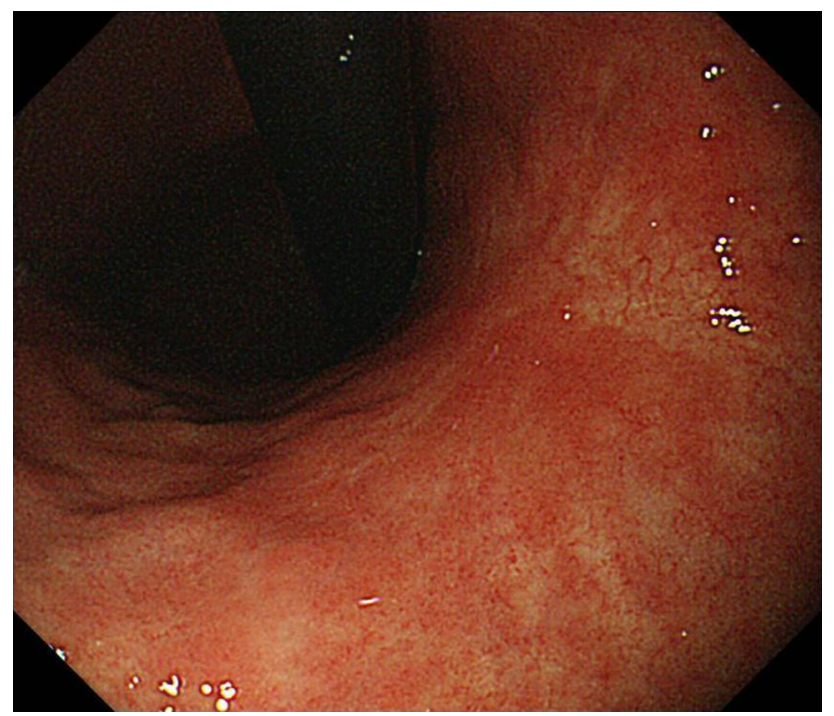

Fig. 2 Follow-up esophagogastroduodenoscopy in December 2010 showed only ulcer scar at his previous site of endoscopic submucosal dissection (ESD)

fulfilled the expanded pathological criteria and was diagnosed as a curative resection.

He was followed up by annual esophagogastroduodenoscopy (EGD). Annual computed tomography (CT) or endoscopic ultrasonography (EUS) was also performed for the first 4 years after resection. Every CT or EUS between 2004 and 2008 indicated no evidence of metastasis. A metachronous gastric cancer was found on the posterior wall of the upper gastric body in June 2008 and was again removed by ESD. The resected specimen revealed intramucosal well-differentiated adenocarcinoma, $17 \times 14 \mathrm{~mm}$ in size, negative for lymphovascular invasion or ulceration, as well as tumor-free margins. Helicobacter

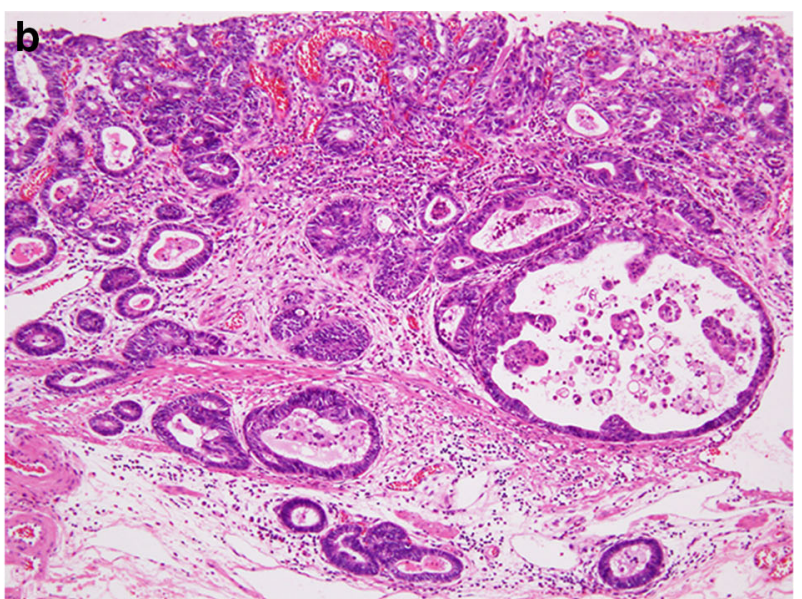

0-IIa lesion without ulceration, $16 \times 8 \mathrm{~mm}$ in size, which had invaded to the submucosa $(100 \mu \mathrm{m}$ from muscularis mucosa) with negative lymphovascular involvement and tumor-free margins

pylori infection was positive and successfully eradicated at that time. The last EGD showed only an ulcer scar at his previous site of ESD in December 2010 (Fig. 2).

In August 2011, the patient underwent EGD for the evaluation of acid reflux and weight loss. The EGD demonstrated irregular ulceration with surrounding wall thickness on the lesser curvature of the middle gastric body, involving the location of the initial ESD scar (Fig. 3a). A biopsy specimen from the ulcerative tumor revealed moderately to poorly differentiated adenocarcinoma (Fig. 3b). We performed a mutation analysis of TP53 using paraffinembedded specimens of two ESDs and the biopsy taken from the ulcerative tumor. Direct sequencing of polymerase chain reaction-amplified deoxyribonucleic acid revealed a somatic nonsense mutation (c. $1024 \mathrm{C}>\mathrm{T}$, p.R342*) in both the initial ESD and the ulcerative tumor (Fig. 4). CT showed a thickened gastric wall, multiple enlarged lymph nodes including the paraaortic node, right adrenal gland swelling, and ascites (Fig. 5). These findings were suggestive for local recurrence and distant metastasis, considered to be unresectable. Thus, he received systemic chemotherapy, but CT indicated progression of peritoneal dissemination and positron emission tomography indicated multiple bone metastasis. Finally, he died of gastric cancer 23 months after recurrence.

\section{Discussion}

Gotoda et al. and Hirasawa et al. reported that no surgical EGC cases without lymphovascular involvement had lymph node metastases in intramucosal differentiated-type cancers $>2 \mathrm{~cm}$ in size without ulceration $(0 / 493,95 \%$ CI $0-0.6 \%$ ), intramucosal differentiated-type cancers $\leq 3 \mathrm{~cm}$ 


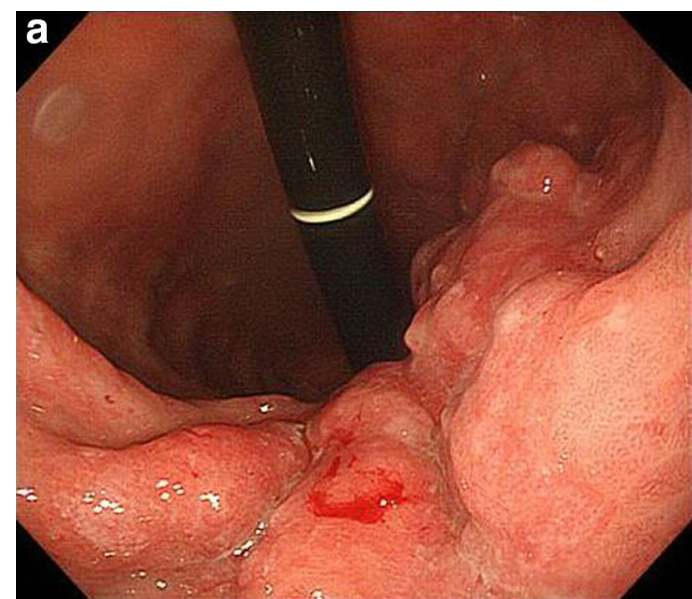

Fig. 3 a Esophagogastroduodenoscopy for the evaluation of acid reflux and weight loss in August 2011 demonstrated irregular ulceration with surrounding wall thickness on the lesser curvature

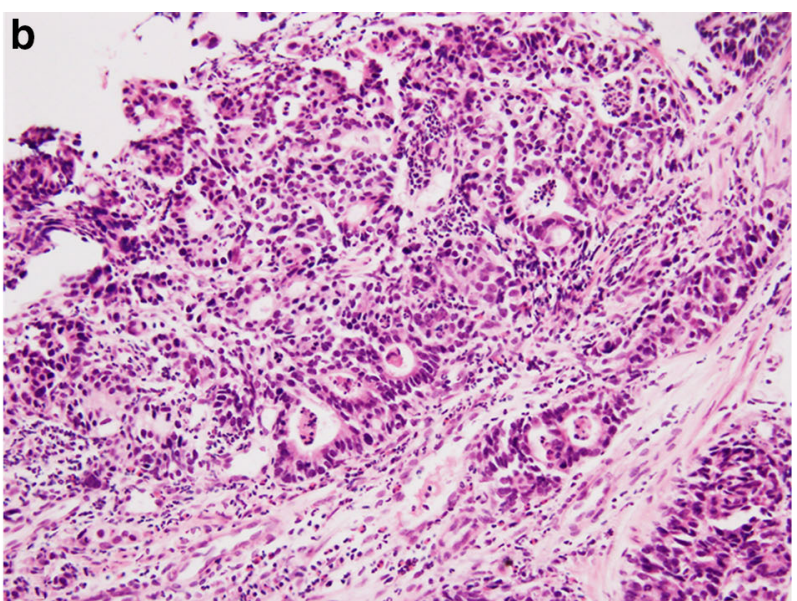

of the middle gastric body, involving the location of the initial ESD scar. b A biopsy specimen from the ulceration revealed moderately to poorly differentiated adenocarcinoma
Fig. 4 A mutation analysis of TP53 identified a single base substitution leading to a premature stop codon in both the initial ESD and biopsy specimen

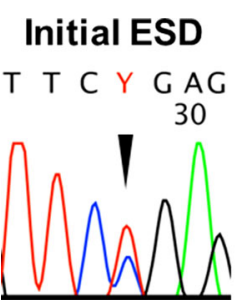

c.1024C>T in size with ulceration $(0 / 488,95 \%$ CI $0-0.6 \%)$, intramucosal undifferentiated-type cancer $\leq 2 \mathrm{~cm}$ in size without ulceration (0/310, $95 \%$ CI $0-0.96 \%)$; and SM1 differentiated-type cancers $\leq 3 \mathrm{~cm}$ in size $(0 / 145,95 \%$ CI $0-2.6 \%$ ) [1, 2]. This result developed into the current expanded pathological criteria for curative endoscopic resection of EGC [3].

ESD can achieve en bloc resection even for large or ulcerative lesions and allows detailed pathological evaluation [4]. The Japanese gastric cancer treatment guidelines (2010, ver. 3) stated that endoscopic resection for these tumors was regarded as an investigational treatment and ESD should be employed [3]. Excellent long-term outcomes of ESD for EGC that fulfilled the foregoing criteria were reported [5-12]. Although these were retrospective studies, the expanded pathological criteria have been considered acceptable.

On this favorable trend of ESD for EGC, we experienced for the first time a fatal case of local recurrence and distant metastasis after curative ESD for SM1 gastric cancer. Expert pathologists reexamined the initial ESD specimen using deeper cuts of the paraffin block to assess for undetectable noncurative factors. This reexamination did not reveal any histological noncurative factors such as an undifferentiated-type adenocarcinoma component in the
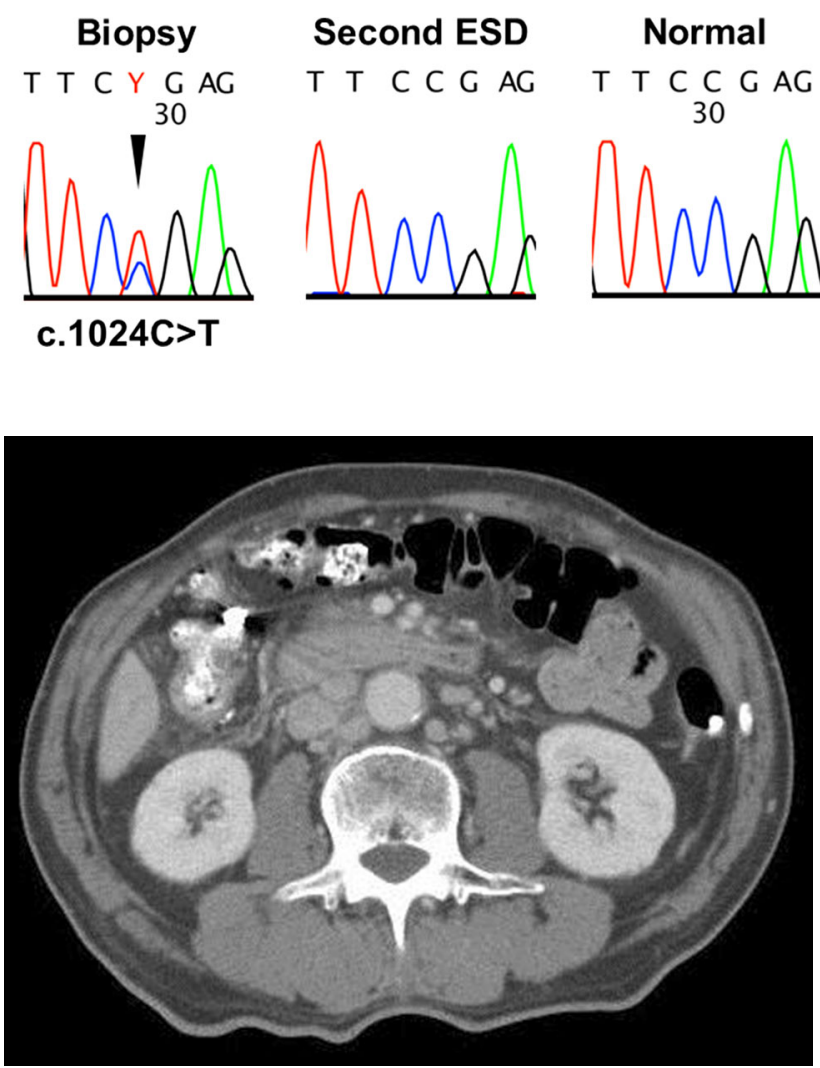

Fig. 5 Computed tomography showed multiple enlarged paraaortic lymph nodes

submucosal layer, lymphovascular invasion, or deeper submucosal invasion.

This case had local recurrence after ESD although the resected specimen indicated a histologically tumor-free margin. One possible explanation is that there might have been lymphovascular invasion or deeper submucosal 
invasion apart from the margin of the initial resected specimen. This growth pattern is not common but it might contribute to the development of a local recurrence. The last EGD 8 months ago indicated no malignancy, and this irregular ulceration was demonstrated just on the initial ESD scar. Furthermore, a mutation analysis of TP53 using paraffin-embedded specimens of the initial ESD and a biopsy taken from the ulcerative tumor revealed the same nonsense mutation. This common mutation was not found in the second ESD. These findings strongly suggested local recurrence after the initial curative ESD rather than metachronous gastric cancer or metastatic tumor that originated from the second ESD.

Oya et al. [13] reported another case of regional lymph node metastasis 49 months after ESD in a patient with SM1 gastric cancer that fulfilled the expanded criteria. The patient's ESD was actually a noncurative resection based on the current version of the treatment guidelines because of a poorly differentiated component with ulceration [3].

The incidence of lymph node metastasis in patients with differentiated-type SM1 gastric cancer with negative lymphovascular involvement $\leq 3 \mathrm{~cm}$ in size had been recognized as $0 \%$, but the upper limit of $95 \% \mathrm{CI}$ in the previous study in surgical cases was $2.6 \%$ [1]. The risk of lymph node metastasis for such SM1 gastric cancer could be slightly higher than for the expanded criteria for intramucosal cancer with a $95 \%$ CI upper limit of $0.6-0.96 \%$. Oda et al. [14] reported a multicenter questionnaire study on long-term outcomes of curative ESD resection for expanded indication in 1,289 patients with EGC. In this study, the percentages of lymph nodes or distant metastasis with more than 5-year follow up was $0.15 \%$ in intramucosal differentiated-type cancers $>2 \mathrm{~cm}$ in size without ulceration $(1 / 708$; $95 \% \mathrm{CI}, 0.004-0.84 \%), 0 \%$ in intramucosal differentiated-type cancers $\leq 3 \mathrm{~cm}$ in size with ulceration $(0 / 305$; $95 \% \mathrm{CI}, 0-1.26 \%), 0 \%$ in intramucosal undifferentiated-type cancer $2 \mathrm{~cm}$ in size without ulceration $(0 / 58 ; 95 \% \mathrm{CI}, 0-6.38 \%)$, and $1.0 \%$ in SM1 differentiated-type cancers $\leq 3 \mathrm{~cm}$ in size $(2 / 218 ; 95 \% \mathrm{CI}$, 0.12-3.55\%: one of the two cases is this case and the other was published by Oya et al. [13]). This retrospective study also indicated the slightly higher $95 \%$ CI upper limits in SM1 differentiated-type cancers $\leq 3 \mathrm{~cm}$ than those in differentiated-type intramucosal gastric cancers, except for the small number of undifferentiated-type intramucosal EGC. Careful follow-up is essential after curative endoscopic resection for the expanded pathological criteria, especially in SM1 differentiated-type gastric cancers.

The expanded pathological criteria are believed to be reasonable because of the favorable long-term outcomes of curative ESD [5-12]. However, all data were retrospective. A few prospective studies were introduced to evaluate the short-term and long-term outcomes of ESD for EGC in
Japan [15-17]. JCOG0607 includes patients who had clinically diagnosed differentiated-type intramucosal cancers $>2 \mathrm{~cm}$ in size without ulceration or $\leq 3 \mathrm{~cm}$ in size with ulceration [15]. The sample size is 470 with 5 years of follow-up. Because the estimated number of the EGC subsequently diagnosed as differentiated-type SM1 gastric cancer $\leq 3 \mathrm{~cm}$ by pathological assessment is expected to be small, this study may not indicate enough evidence for such SM1 cancers. J-WEB/EGC includes preoperative absolute indication as well as expanded indication, and approximately 10,000 patients will be enrolled by 41 institutions for 2 years. The result of this study may indicate the validity of all expanded pathological criteria in future [16].

This case had local and distant recurrence 74 months after initial ESD. Because recurrence of gastric cancer generally occurs within 5 years in surgical cases and there was no report of recurrence after curative gastric ESD at that time, surveillance CT or EUS was not performed after 2008 [18]. However, Choi et al. [19] analyzed the timing of the recurrence after curative gastrectomy and concluded that early stage and differentiated histological type were significantly associated with late recurrence. In addition, $H$. pylori eradication might affect late recurrence because that could suppress the growth of gastric cancer [20]. Therefore, careful follow-up longer than 5 years by $\mathrm{CT}$ as well as annual EGD may be needed for the patients with EGC that was treated by ESD and met the expanded pathological criteria.

In conclusion, we showed a fatal case of local recurrence and distant metastasis following curative ESD of EGC. The results of a prospective study are warranted to determine whether the expanded pathological criteria can include differentiated-type SM1 gastric cancers.

Conflict of interest None.

\section{References}

1. Gotoda T, Yanagisawa A, Sasako M, Ono H, Nakanishi Y, Shimoda $\mathrm{T}$, et al. Incidence of lymph node metastasis from early gastric cancer: estimation with a large number of cases at two large centers. Gastric Cancer. 2000;3:219-25.

2. Hirasawa T, Gotoda T, Miyata S, Kato Y, Shimoda T, Taniguchi $\mathrm{H}$, et al. Incidence of lymph node metastasis and the feasibility of endoscopic resection for undifferentiated-type early gastric cancer. Gastric Cancer. 2009;12:148-52.

3. Japanese Gastric Cancer Association. Japanese gastric cancer treatment guidelines 2010 (ver. 3). Gastric Cancer. 2011;14: 113-23.

4. Oda I, Saito D, Tada M, Iishi H, Tanabe S, Oyama T, et al. A multicenter retrospective study of endoscopic resection for early gastric cancer. Gastric Cancer. 2006;9:262-70.

5. Isomoto H, Shikuwa S, Yamaguchi N, Fukuda E, Ikeda K, Nishiyama $\mathrm{H}$, et al. Endoscopic submucosal dissection for early 
gastric cancer: a large-scale feasibility study. Gut. 2009;58: 331-6.

6. Goto O, Fujishiro M, Kodashima S, Ono S, Omata M. Outcomes of endoscopic submucosal dissection for early gastric cancer with special reference to validation for curability criteria. Endoscopy. 2009;41:118-22.

7. Ahn JY, Jung HY, Choi KD, Choi JY, Kim MY, Lee JH, et al. Endoscopic and oncologic outcomes after endoscopic resection for early gastric cancer: 1370 cases of absolute and extended indications. Gastrointest Endosc. 2011;74:485-93.

8. Gotoda T, Iwasaki M, Kusano C, Seewald S, Oda I. Endoscopic resection of early gastric cancer treated by guideline and expanded National Cancer Centre criteria. Br J Surg. 2010; 97:868-71.

9. Toyonaga T, Man-i M, East JE, Nishino E, Ono W, Hirooka T, et al. 1,635 Endoscopic submucosal dissection cases in the esophagus, stomach, and colorectum: complication rates and long-term outcomes. Surg Endosc. 2013;2013(27):1000-8.

10. Kosaka T, Endo M, Toya Y, Abiko Y, Kudara N, Inomata M et al. Long-term outcomes of endoscopic submucosal dissection for early gastric cancer: a single-center retrospective study. Dig Endosc 2013 Apr. doi:10.1111/den.12099. [Epub ahead of print].

11. Tanabe S, Ishido K, Higuchi K, Sasaki T, Katada C, Azuma M et al. Long-term outcomes of endoscopic submucosal dissection for early gastric cancer: a retrospective comparison with conventional endoscopic resection in a single center. Gastric Cancer 2013 Apr 11. [Epub ahead of print].

12. Abe S, Oda I, Suzuki H, Nonaka S, Yoshinaga S, Odagaki T, et al. Short- and long-term outcomes of endoscopic submucosal dissection for undifferentiated early gastric cancer. Endoscopy. 2013;45:703-7.

13. Oya H, Gotoda T, Kinjo T, Suzuki H, Yoshinaga S, Taniguchi H, et al. A case of lymph node metastasis following a curative endoscopic submucosal dissection of an early gastric cancer. Gastric Cancer. 2012;15:221-5.
14. Oda I, Oyama T, Abe S, Ohnita K, Kosaka T, Hirasawa K, et al. Preliminary results of multicenter questionnaire study on longterm outcomes of curative endoscopic submucosal dissection for early gastric cancer. Dig Endosc 2013 Jul 5. doi:10.1111/den. 12141. [Epub ahead of print].

15. Kurokawa Y, Hasuike N, Ono H, Boku N, Fukuda H, Gastrointestinal Oncology Study Group of Japan Clinical Oncology Group. A phase II trial of endoscopic submucosal dissection for mucosal gastric cancer: Japan Clinical Oncology Group Study JCOG0607. Jpn J Clin Oncol. 2009;39:464-6.

16. Oda I, Shimazu T, Ono H, Tanabe S, Iishi H, Kondo H, et al. Design of Japanese multicenter prospective cohort study of endoscopic resection for early gastric cancer using Web registry (J-WEB/EGC). Gastric Cancer. 2012;15:451-4.

17. Takizawa K, Takashima A, Kimura A, Mizusawa J, Hasuike N, Ono H, Gastrointestinal Endoscopy Study Group (GIESG) of Japan Clinical Oncology Group (JCOG), Stomach Cancer Study Group (SCSG) of Japan Clinical Oncology Group (JCOG), et al. A phase II clinical trial of endoscopic submucosal dissection for early gastric cancer of undifferentiated type: Japan Clinical Oncology Group study JCOG1009/1010. Jpn J Clin Oncol. 2013;43:87-91.

18. Feng XY, Chen YB, Wang W, Guan YX, Li YF, Chen S, et al. Time-varying pattern of recurrence risk for gastric cancer patients. Med Oncol. 2013;30:514

19. Choi JY, Ha TK, Kwon SJ. Clinicopathologic characteristics of gastric cancer patients according to the timing of the recurrence after curative surgery. J Gastric Cancer. 2011;11:46-54.

20. Sasaki A, Kitadai Y, Ito M, Sumii M, Tanaka S, Yoshihara M, et al. Helicobacter pylori infection influences tumor growth of human gastric carcinomas. Scand J Gastroenterol. 2003;38: $153-8$. 